Archives de sciences sociales des religions

172 | octobre-décembre

Bulletin Bibliographique

François Trémolières, Approches de l'indicible. Études bremondiennes

Grenoble, Jérôme Millon, 2014, 264 p.

Frédéric Gugelot

CpenEdition

Journals

Édition électronique

URL : http://journals.openedition.org/assr/27481

DOI : $10.4000 /$ assr. 27481

ISSN : $1777-5825$

Éditeur

Éditions de l'EHESS

Édition imprimée

Date de publication : 1 octobre 2015

Pagination : 384

ISBN : 978-2-7132-2515-4

ISSN : 0335-5985

Référence électronique

Frédéric Gugelot, «François Trémolières, Approches de l'indicible. Études bremondiennes », Archives

de sciences sociales des religions [En ligne], 172 I octobre-décembre, mis en ligne le 22 juin 2016, consulté le 24 septembre 2020. URL : http://journals.openedition.org/assr/27481 ; DOI : https:// doi.org/10.4000/assr.27481

Ce document a été généré automatiquement le 24 septembre 2020

(C) Archives de sciences sociales des religions 


\section{François Trémolières, Approches de l'indicible. Études bremondiennes}

Grenoble, Jérôme Millon, 2014, 264 p.

Frédéric Gugelot

\section{RÉFÉRENCE}

François Trémolières, Approches de l'indicible. Études bremondiennes, Grenoble, Jérôme Millon, 2014, 264 p. 
1 Heureuse et excellente initiative que cette publication qui réunit plusieurs des textes écrits par François Trémolières autour d'Henri Bremond, d'autant plus que l'ouvrage rend accessible un ensemble très épars d'articles.

2 Touche après touche, sans que ce soit impressionniste et en s'appuyant sur une érudition sans faille, l'auteur parvient à cerner l'immense projet de l'Histoire littéraire du sentiment religieux en France depuis la fin des guerres de Religion jusqu'à nos jours dont François Trémolières est le

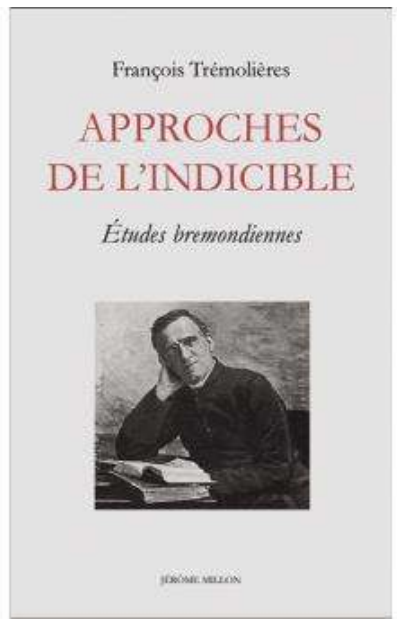
maitre d'œuvre de la réédition scientifique réalisée en 2006. Ce livre rejoint tout un ensemble d'autres publications assurées par Jérôme Millon qui, de l'œuvre de Bremond aux recherches qu'elle a suscitées, permettent de cerner le contexte de sa publication et de sa réception. Ces dix textes dégagent l'avant, le pendant et l'après de «l'entreprise bremondienne » (p. 63).

Trémolières restitue dans son contexte (foi mystique et raison critique) mais aussi dans sa postérité (Michel de Certeau, Émile Goichot) cette réalisation. En restaurant tout un pan disparu de la production du XVII ${ }^{\mathrm{e}}$ siècle, qui se réduisait jusque-là largement, sous l'influence de Sainte-Beuve, à Port-Royal, Bremond montre le bourgeonnement de la littérature d'inspiration chrétienne. Le fait essentiel du siècle des saints est « la tension entre la conscience et l'institution [qui] traumatise littéralement l'expérience chrétienne à partir du moment où la société entière cesse d'être un langage religieux ». L'abbé a rendu ses lettres de noblesse à toute une littérature religieuse dont il postule qu'elle livre accès à l'expérience spirituelle et dont il dévoile la réelle affinité de leur objet, la mystique, avec la littérature. Ce qui est au cœur est cette relation de l'expérience au discours, le fait que « la mystique se constitue comme expérience, par son irréductibilité au discours » (p. 13).

4 L'auteur dessine avec justesse une "situation de Bremond ", titre d'un des chapitres. Jésuite de formation, il est contraint de quitter discrètement la Compagnie en 1904 certainement à la suite de ses engagements et sympathies avec les modernistes. Il ne quittera jamais l'Église et n'abandonne pas la prêtrise. Il est un « clerc » (p. 100) soumis à toutes les décisions de sa hiérarchie, demandant à chaque parution l'imprimatur (p. 213). C'est qu'il préserve une vision du religieux, qui, au-delà des religions historiques, position pas si éloignée de celle de Loisy, laisse toute sa place à la foi (p. 102). L'expérience spirituelle l'emporte à ses yeux sur toute formulation dogmatique, toute canonisation par l'Église (p. 125). Bremond réaffirme la « réalité de la foi » mais il souhaite en dépasser les expressions dogmatiques pour renouer avec l'expérience religieuse, le «sentiment». Ce qui lui permet de dépasser le piège "moderniste» de la question de l'historicité du dogme (p.31). Sa postérité est indéniable. «L'expérience individuelle serait une chose saisissable derrière les textes. En réalité, n'est lisible, de chaque expérience spirituelle, que ce qui en est reçu. Et ce qui en est reçu à tel moment, c'est un état du texte ", note de M. de Certeau, dans L'absent de l'histoire (p. 32). 
5 Mais le «modernisme" hante l'œuvre de Bremond, qui appartient à ces savants catholiques qui autour $\mathrm{du} \mathrm{XIX}^{\mathrm{e}}$ et $\mathrm{xx}^{\mathrm{e}}$ siècle travaillent à trouver une réponse catholique au développement de sciences des Écritures autonomes de l'institution ecclésiale. Ils aspirent à réformer de l'intérieur l'institution, sans idée de rupture. Cette tentative est brisée par l'Église elle-même consciente des risques d'une telle tentative. Bremond croit possible pour l'écrivain de rendre témoignage du monde invisible, de cerner les expériences mystiques, religieuses et poétiques entremêlées au risque de les confondre (p.147). Bremond note qu'il est impossible dans un hymne de distinguer «ce qui est poésie de ce qui est prière» (p.148). L'accueil réservé à l'œuvre de l'abbé montre l'inquiétude de l'institution quant à une possible laïcisation des approches (p. 95-99). Bremond a pourtant choisi de se replier sur le champ de la spiritualité française du XVII ${ }^{\mathrm{e}}$ siècle, moins exposé que l'histoire des dogmes, l'histoire de l'Église ou l'exégèse. Il ramène au jour cette littérature dévote et ces écrits spirituels largement oubliés. Cette prudence est amplifiée par son choix de se cantonner aux œuvres catholiques, n'abordant pas la production d'origine protestante. L'auteur insiste sur ce «souci d'orthodoxie» (p.114). Néanmoins le projet de Bremond par sa nature même décloisonne, "déconfessionnalise ", s'interroge l'auteur (p.118), l'histoire de la spiritualité classique.

6 Les travaux de Bremond s'inscrivent dans une "vague mystique », un réveil de la spiritualité qui correspond à un transfert de l'intellectuel au spirituel (p.78), un déplacement vers «la prière au détriment de la réflexion» (E. Fouilloux, cité p. 83). Cette préférence exprimée envers l'amour de Dieu plus qu'envers l'intellectualisme chrétien résonne jusque dans son rejet de l'esprit de système et son incompréhension des positions de Maurras par trop de l'ordre de la raison (p. 90). Bremond n'est pas sensible aux sirènes de l'Action française. De même bien qu'historien littéraire, il se méfie des liens entre littérature et religion (p. 137). Il s'appuie sur le développement de la «psychologie religieuse (W. James, H. Delacroix, etc.)» (p.141) pour recevoir la littérature comme mode de connaissance de la nature de l'homme. Ceci lui permet d'appréhender ce pan de la littérature comme objet d'études, il est vrai, en le réduisant largement à une littérature de témoignage : «tant de pages qui nous attestent sinon l'histoire exacte de tel martyr, du moins la foi vivante des générations qui se sont transmises et ont embelli cette histoire » (p. 143).

7 Le livre cerne la réception de l'œuvre, tant du vivant de l'auteur qu'ultérieurement. Déjà parce que «l'écriture pose un problème à tout prêtre " (André Blanchet, p. 135). Les textes consacrés à la mise à l'index de la sainte Chantal, une hagiographie de Bremond publiée en 1912, démontrent le rôle des dénonciations issues directement de la Visitation (p. 199), qui après hésitation rejette la lecture par Bremond de la figure fondatrice. Ainsi celui-ci ferait de l'union spirituelle entre Jeanne de Chantal et François de Sales une simple amitié naturelle: «C'est bien là, pris sur le vif, l'esprit moderniste qui consiste à vider l'histoire de l'Église de l'élément surnaturel », dénonce un journal local (p. 156). Ces accusations voient leur impact démultiplié grâce à l'appel à un théologien qui forge les arguments même de la condamnation. On reproche à Bremond une "étude psychologique " des personnages, une écriture où l'érudition prend le pas sur l'édification que se doit d'être toute vie de saint. L'autonomie d'un prêtre auteur semble impossible. La condamnation s'appuie sur une lecture fine des ouvrages, mais aussi sur une grille de lecture qui justifie son sort. L'évaluateur du livre, le Père Lémius, est celui-là même qui aurait rédigé la partie doctrinale de l'encyclique 
de condamnation du modernisme, Pascendi. Cela lui donne une autorité supplémentaire quand il accuse un ouvrage d'être "moderniste ", mais prouve à quel point la vision préfigurée du lecteur va conduire son travail. La traduction du passionnant rapport à l'origine de la condamnation montre une étude très approfondie du texte, des termes choisis, en s'appuyant sur une sélection de citations (p. 179-193). Au fond, le primat de l'expérience religieuse "revient à historiciser le dogme et à relativiser les autres sources de foi: la tradition, l'autorité» (p.168): "On sait que modernisme et hypercritique vont de pair, et que pour les hypercritiques la tradition n'a pas de valeur, car à leurs yeux seuls les documents en possèdent » (extrait du rapport de la Sacrée Congrégation de l'Index, p. 179). L'expérience Sainte Chantal convainc Bremond du piège du genre hagiographique. Son livre est dénoncé comme exemplaire du «style moderniste" tout comme Le Saint de Fogazzaro est condamné en 1906 comme l'expression romanesque du "modernisme littéraire » (p. 174). L'institution mêle donc doctrine et esthétique (p. 218). Puisque la littérature touche à la morale, du point de vue de l'Église, celle-ci ne peut légitimement intervenir dans le jugement littéraire. La question de l'autonomie du créateur est une fois de plus posée surtout quand il s'agit d'un prêtre : «Et tout cela écrit dans une vie de saint écrite par un prêtre! », conclut le rapport de l'Index (p. 193). Les querelles littéraires ne sont pas spirituellement neutres. On comprend mieux au vu de la condamnation de 1913 le choix de Bremond et la réussite de son projet. Ainsi l'auteur consacre un texte aux apports d'Émile Goichot, pour qui l'Histoire littéraire fut peut-être la seule entreprise moderniste qui ait réussi puisqu'elle a été «écrite dans l'Église, a été reçue par l'Église » (p. 129). Ces quelques lignes extraites de l'Almanach catholique français pour 1920, publiées sous le patronage du Comité catholique de propagande française à l'étranger par Bloud et Gay, en témoigne : «Un autre ouvrage, de bien plus grande envergure encore, et d'un mérite tout autrement exceptionnel, c'est l'Histoire littéraire du sentiment religieux en France [...]. Rarement vous aurez fait lecture plus réjouissante et plus captivante, non moins qu'instructive et édifiante. » Et on comprend l'incompréhension qui rendit possible l'entreprise.

8 Au-delà des aléas de l'œuvre de l'abbé Bremond, l'auteur dessine, texte après texte, l'enjeu essentiel pour le catholicisme dans son rapport à la modernité, de cette œuvre érudite consacrée aux auteurs mystiques et dévots du xvII siècle. Il ne s'agit pas uniquement de les ramener à la lumière du jour, mais, aussi, à travers eux, d'offrir à la foi, une présence au cœur $\mathrm{du} \mathrm{xx}^{\mathrm{e}}$ siècle. S'y découvrent les contraintes, les difficultés, les échecs et les réussites d'un projet de réforme intérieure de l'Église qui, bien avant Vatican II, espérait renouer avec son temps. Si l'Histoire littéraire, bien qu'inachevée, s'est imposée, la réforme s'est estompée. Le projet semble avoir avalé l'homme au point que les espérances du départ se sont noyées dans les multiples volumes et les contraintes d'une écriture sous surveillance. 